American Journal of Infectious Diseases 4 (3): 183-186, 2008

ISSN 1553-6203

(C) 2008 Science Publications

\title{
Multiple Pulmonary and Multivesicular Interatrial Septum Hydatid Cysts in A Native Italian Patient
}

\author{
${ }^{1}$ A. Romano, ${ }^{1}$ S. Giordano, ${ }^{2}$ F. Follis, ${ }^{3}$ L. Marasa, ${ }^{4}$ A. Mazzola, ${ }^{5}$ D. Cabibbi and ${ }^{4}$ P. Di Carlo \\ ${ }^{1}$ U.O. Malattie Infettive ARNAS Civico, Palermo, Italy \\ ${ }^{2}$ U.O. Cardiochirurgia adulti ARNAS Civico, Palermo, Italy \\ ${ }^{3}$ U.O. Anatomia Patologica ARNAS Civico, Palermo, Italy \\ ${ }^{4}$ Istituto di Patologia Infettiva e Virologia, Università di Palermo, Italy \\ ${ }^{5}$ Dipartimento di Patologia Umana, Università Degli Studi, Palermo
}

\begin{abstract}
Multivesicular, pulmonary and cardiac hydatidosis are rarely observed and can give rise to serious complications. Cysts can remain asymptomatic for a long time, until they reveal themselves perforating into cardiac chambers and/or pulmonary arteries or the systemic circulation. A rare case of multivesicular interatrial septum hydatid cyst with multiple pulmonary involvement in a native Italian farm labourer is reported. Clinical, radiological, serological and histological findings are described. MR imaging showed the exact anatomic location and the multivesicular nature of the cardiac cyst and was useful in planning surgical treatment. A successful outcome was achieved with a combination of pre- and post-operative albendazole therapy and a three-step surgery procedure. The patient made a rapid recovery and his post-operative period was totally uneventful. This case highlights the importance of an early multidisciplinary surgical approach and long-term chemotherapy treatment of this serious and rare disease. MR Imaging was crucial in the pre-surgical and follow-up observations.
\end{abstract}

Key words: Echinococcus granulosus, cardiac hydatidosis, multivesicular hydatic cyst, pulmonary hydatidosis

\section{INTRODUCTION}

Echinococcosis is a zoonotic disease caused by the ova of the Echinococcus granulosus parasite ${ }^{[1-2]}$. The disease is encountered in most parts of the world and occurs most commonly in sheep farming and cattle farming areas of South America, New Zealand, the Philippines, China, Arabia, Eastern Europe and the Mediterranean basin ${ }^{[3-7]}$.

The parasite is a small Taenia (less than $1 \mathrm{~cm}$ long). Dogs, wolves and other carnivorous animals are the definitive hosts of its adult form. Ova are excreted in the animals' faeces and contaminate herbage, remaining viable for weeks in cool, damp places. When viable ova are ingested by herbivores, they develop into unilocular cysts in the viscera, in the liver and lungs in particular. The initial lesion is a fluid-filled, cystic structure consisting of three layers: the outermost pericyst composed of modified host cells and fibrous tissue; a middle laminated membrane resembling eggwhite and a thin inner germinal membrane. Hydatid cysts progressively enlarge and daughter cysts develop within the cavity of the large parent cyst (multivesicular cyst). When the nourishing hydatid fluid is replaced by formed elements the lesion dies and eventually becomes calcified and biologically inert ${ }^{[1,2,8]}$.

Human infection is acquired by eating raw meat or vegetables containing ova. Ingested ova burrow through the wall of the intestine, enter the portal circulation system and then pass on to the liver. About 10 to 20 percent of ova may migrate from the liver to vessels in the lung.

The most common target of the Echinococcus is the liver (60\%), followed by the lung (20-30\%). Any other localization is extremely rare, even in endemic areas: cardiac involvement is reported in less than 3\% of cases, brain 1-3\%, kidney 2-3\%, pancreas $0.19-2 \%$, breast $0.27 \%$ and muscle $3 \%$. In any case, involvement of all these different sites is related to cyst rupture with scolex dissemination into the blood flow ${ }^{[1,2,9,10]}$.

Once larvae invade myocardial tissue through the coronary circulation, they need about $1-5$ years to develop into their adult form, sometimes infestation can lead to multiorgan involvement ${ }^{[11]}$.

We report a rare case of multiple pulmonary and inter atria multivescicular hydatic cysts in a native Sicilian patient.

Corresponding Author: Daniela Cabibi, Dipartimento di Patologia Umana, Università degli Studi, Palermo Via del Vespro, 129 Palermo Tel: +39 0916553533 Fax: +39 0916553549 


\section{CASE REPORT}

A 59 year-old man, working in a Sicilian rural area, with no significant clinical history, consulted his general physician for a persistent and worsening cough. Blood chemistry values were all normal. Chest radiography showed multiple round opacities without reaction in the lung tissue, indicative of lung hydatidosis and an enlarged cardiac silhouette. Twodimensional echocardiography revealed a multicompartmented cystic mass adhering to the wall of the right atrium, $5 \times 4 \mathrm{~cm}$. The electrocardiogram was normal.

In preparation for surgery, the patient was hospitalized and started on a treatment with Albendazole $10 \mathrm{mg} / \mathrm{kg} /$ day.

Cardiac magnetic resonance (MR) revealed the multivescicular nature of the cyst, packed with daughters cysts and showed its inter atrial localization (Fig. 1). Extended check-up, abdominal MR, cranial MR and skeleton X-ray did not reveal any additional cysts.

The ELISA test for Echinococcus granulosus showed the presence of $\operatorname{IgG}$ and $\operatorname{IgE}$ specific antibodies. The patient's pre-operative biochemical parameters revealed moderate anaemia $\left(7.8 \mathrm{mg} \mathrm{dL}^{-1}\right)$ and mild increase of transaminases (GOT max. value $131 \mathrm{UI} / \mathrm{L})$.

Median sternotomy was the procedure used and the patient was operated on using cardiopulmonary bypass. A heart incision was performed to expose the hydatid cyst. This was covered with wet sponges to prevent embolism and the inoculation of scolex in to other cardiac structures. During the operation, a cystic mass measuring $5 \times 4$ centimetres, originating in the interatrial septum and multivesicular in form, was excised and extirpated.

A histopathological examination confirmed the diagnosis of hydatid cyst revealing a multilocular cyst with an outer laminated chitinous layer, an inner germinal layer and a granulomatose reaction of the host, with focal necrosis (Fig. 2A).

All the patient's biological parameters had returned to normal one week after surgery and he was discharged from hospital with the recommendation to continue the Albendazole therapy.

Forty days after the first observation, the patient was readmitted to hospital to remove the lung cysts.

Surgical exeresis of the lung upper left lobe was performed and about 20 cysts were removed. Even in this case, histopathological analysis confirmed Echinococcus aetiology, revealing multiple cysts with with a laminated chitinous layer, but a fibrous

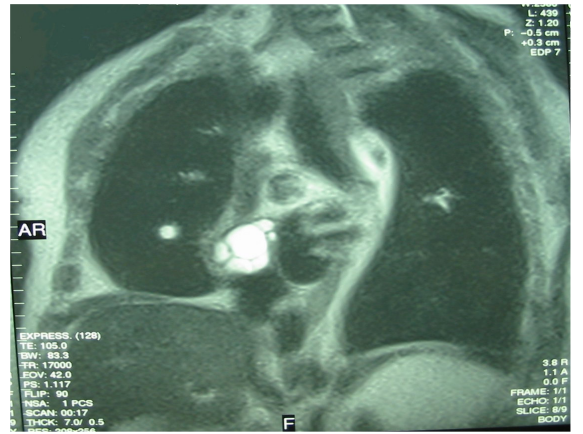

Fig. 1: MR oblique scan showing a cardiac multivesicular hydatic cyst

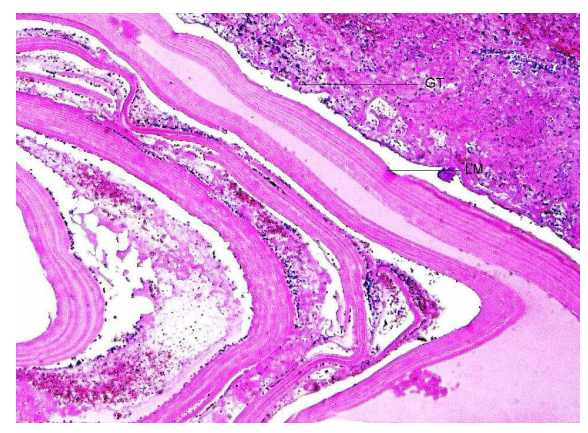

Fig. 2A: Histologic section of right atrium showing laminated membranes of the parasite and granulomatose inflammation tissue of the host. PAS stain, overall magnification 100x

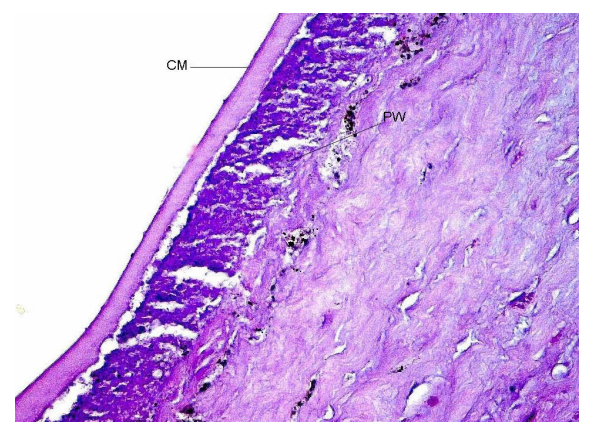

Fig. 2B: Histologic section of lung showing the laminated membrane of the parasite surrounded by a thin necrotic layer and by the fibrous reaction of the lung parenchyma PAS stain, overall magnification 100x

pericystium was absent and the cyst was surrounded by a thin layer of necrotic tissu and by the fibrous reaction of the lung parenchyma. Figure 2B Two weeks after this second operation, the chest radiography revealed a clear improvement of the thoracic lesion. 
Subsequently, the patient was again hospitalized for the third and final surgical operation to remove cysts from the lung upper right lobe. During the postoperative period a gas flow from pleural drainage occurred and consequently a unidirectional Heimlich valve was employed, with full recovery.

No problems were encountered during the one-year post-surgical follow-up period.

\section{DISCUSSION}

Hydatidosis is rarely reported in the native European population, as most cases involve immigrants from countries where the disease is endemic; cases of disease localized within the inter atrial septum are exceptional $^{[3-7,12-14]}$.

Studies have reported many differences in parasite characteristics among geographic strains due to local patterns of transmission. In Sicily, cystic Echinococcosis was found in $67.1 \%$ of cattle, with a fertility of $4 \%$ and in $57.6 \%$ of sheep, with $9.2 \%$ of viable cysts. Biomolecular investigations have found G1 strain in sheep and cattle ${ }^{[7]}$.

In our patient, hydatidosis was manifested by a persistent cough and cardiac involvement was suggested by chest roentgenograms: generally, cardiac echinococcosis is asymptomatic and symptoms appear as a result of myocardial pressure (myocardial ischemia, rhythm and conduction abnormalities, cardiac arrest) due to cyst enlargement or rupture (anaphylactic shock or systemic embolization) $)^{[15-17]}$.

Transthoracic echocardiography, TC and MR can be successfully employed in cardiac hydatidosis diagnosis; other more invasive techniques are available, but the risk related to cyst rupture could be high, possibly resulting in anaphylactic shock or systemic contamination. In the patient described here, cardiac MR imaging was the most reliable technique to identify cyst findings and anatomical connection with cardiac tissue $^{[18,19]}$.

Diagnosis and appropriate surgical excision of pulmonary and cardiac cysts prevented potentially lethal complications, which are more likely in cases of fertile forms such as multivesicular cysts with daughter cysts. Typical cardiac hydatic cysts are univesicular and reports of multivesicular cysts are rare. Univesicular cysts have a laminated membrane that should be removed along with the cyst fluid. For multivesicular cysts, the gelatinous cyst content and numerous daughter cysts must be scooped out. Evacuation of cyst content must be meticulous, ensuring no parasitic material remains ${ }^{[8,14,20,21]}$.

Although superficially located cysts can be successfully removed with the beating-heart technique, resection under cardiopulmonary bypass is considered the safest method, as it carries the least risk of cyst content spillage during the procedure ${ }^{[22]}$.

Our patient underwent invasive surgery three times and, in the absence of coexistent risk factors, the postoperative period was totally uneventful.

In uncomplicated cases, surgical resection remains the preferred treatment; however patients with multiple cysts should be treated with albendazole or combined medical treatment ${ }^{[14,19,22,23]}$. In addition, during surgery, special care must be taken to remove the cyst intact, as a leakage of cyst fluid can lead to general toxicity and the dissemination of microscopic fragments can generate new cysts ${ }^{[1,17,20]}$. In our patient, this was prevented by long-term medical treatment with albendazole in the pre- and post-surgery phases.

This case emphasizes the importance of a cardiac involvement investigation in all patients with lung localization, due to the lack of specific symptoms. Management of this kind of patient requires a multidisciplinary approach involving the collaboration of different specialists to guarantee the best diagnostic and therapeutic results.

\section{REFERENCES}

1. King, C., 2000. Cestodes (tapeworms). In: Mandell, G., J. Bennett, R. Dolin (Eds.). Principles and Practices of Infectious Disease. 5th Edn. 633640. Curchill Livingstone, New York.

2. Moro, P., A. Gonzales and R. Gilman, 2000. Cystic hydatid disease. In T. Strickland (Ed.). Hunter's Tropical Medicine and Emerging Disease, (8th Edn.). 866-875. Philadephia: WB Saunders.

3. Craig, P.S. and E. Larrieu, 2006. Control of cystic echinococcosis/hydatidosis: 1863-2002. Adv. Parasitol. 61: 443-508.

4. Moro, P. and P.M. Schantz, 2006. Cystic echinococcosis in the americas Parasitol Int., 55 (suppl): S181-186.

5. Sadjjadi, S.M., 2006. Present situation of echinococcosis in the Middle East and Arabic North Africa. Parasitol. Int., 55 Suppl: S197-202.

6. Yang, Y.R., T. Sun, Z. Li, J. Zhang Teng, X. Liu, R. Liu, R. Zhao, M.K. Jones, Y. Wang, H. Wen, X. Feng, Q. Zhao, Y. Zhao, D. Shi, B. Bartholomot, D.A. Vuitton, D. Pleydell, P. Giraudoux, A. Ito, M.F. Danson, B. Boufana, P.S. Craig, G.M. Williams and D.P. McManus, 2006. Community surveys and risk factor analysis of human alveolar and cystic echinococcosis in Ningxia Hui Autonomous Region, China. Bull World Health Organ, 84 (10): 840. 
7. Garippa, G., 2006. Updates on cystic echinococcosis (CE) in Italy. Parassitologia, 48 (12): $57-59$.

8. Lewall, D.B., 1998. Dec. Hydatid disease: Biology, Pathology, imaging and classification. Clin. Radiol., 53 (12): 863-74.

9. Bhatia, G., 1997. Echinococcus. Semin Respir Infect., 12: 171-187.

10. Abi, F. F. el Fares, D. Khaiz and A. Bouzidi, 1989. Unusual localizations of hydatid cysts. Apropos of 40 cases. J. Chir., 126: 307-312.

11. Aydogdu, T., N. Sahin, V. Ulusan, F. Gurpinar, C. Turkay and O. Bayezid, 2001. Right atrial hydatid cyst associated with multiple organ involvement: Case report. J. Thorac. Cardiovasc Surg., 121: 1009-1011.

12. Mandke, J.V. and V.P. Sanzgiri, 1992. Hydatid cyst of the interatrial and interventricular septum of heart. Chest, 102: 1916-1917.

13. Karabay, O., A. Onen, F. Yildiz, E. Yilmaz, C.A. Erdal, A. Sanli, G. Kilci, I. Algin, O. Itil and U. Acikel, 2004. The case of a cyst hydatid localized within the interatrial septum. Jap. Heart J., 45 (4): 703-707.

14. Adnan Uysalel, Levent Yazicioglu, Atilla Aral and Hakki Akalin, 1998. A multivesicular cardiac hydatid cyst with hepatic involvement. Eur. J. Cardiothorac Surg., 14 (3): 335-337.

15. Malamou-Mitsi, V., L. Pappa, T. Vougiouklakis, D. Peschos, N. Kazakos, G. Grekas, D. Sideris and N.J. Agnantis, 2002. Sudden death due to an unrecognized cardiac hydatid cyst. J. Forensic Sci., 47 (5): 1062-1064.

16. Kaplan, M., M. Demirtas, S. Cimen and A. Ozler, 2001. Cardiac hydatid cysts with intracavitary expansion. Ann. Thorac Surg., 71: 1587-90.
17. Lahdhili, H., S. Hachicha, M. Ziadi and H. Thameur, 2002. Acute pulmonary embolism due to the rupture of a right ventricle hydatic cyst. Eur. J. Cardio-Thoracic Surg., 22: 462-464.

18. Atilgan, D., H. Kudat, T. Tükek, et al., 2002. Role of transesophageal echocardiography in diagnosis and management of cardiac hydatid cyst: Report of three cases and review of the literature. J. Am. Soc. Echocardiogr, 15: 271-274.

19. Fertin, M., F. Mounquet, R. Lallemant, V. Gaxotte, C. Decoene, B. Larrue, E. Dutoit, P. deGroote and G. Fayad, 2006. Diagnosis, imaging and treatment of an unusual cardiac hydatid cyst. Cardiovasc Pathol, 15 (6): 356-358.

20. Tarcoveanu, E., G. Dimofte, C. Bradea, F. Crumpei, R. Anton and R. Moldovanu, 2006. Multiple peritoneal hydatid disease after rupture of a multivesicular hepatic hydatid cyst. Case Report J. Gastrointestin Liver Dis., 15 (3): 301-305.

21. Zerem, E. and R. Jusufovic, 2006. Percutaneous treatment of univesicular versus multivesicular hepatic hydatid cysts Surg. Endosc., 20 (10): 1543-1547.

22. Erentung, V., N. Bozbuga, K. Nirali, I. Matraci, C. Kaymaz et al., 2004. Cardiac Hydatid Cysts: Surgical treatments and results. J. Card. Surg., 19 (4): 358-360.

23. Ayles, H.M., E.L. Corbett, L. Taylor, A.G. Cowie, J. Bligh, K. Walmsley and A.D. Bryceson, 2002. A combined medical and surgical approach to hydatid disease: 12 years' experience at the Hospital for Tropical Diseases, London. Ann. R. Coll Surg Engl., 84 (2): 100-105. 